\title{
Understanding the Formation and Evolution of the Kuiper Belt by Exploring the Haumea System
}

\author{
White Paper Submitted To \\ Decadal Survey in Planetary Science and Astrobiology 2023-2032
}

\begin{abstract}
Submitted By:
Julie Brisset ${ }^{1 *}$, Estela Fernandez-Valenzuela ${ }^{1}$, Amanda Sickafoose ${ }^{2}$, Flaviane Venditti ${ }^{3}$, Akbar Whizin $^{4}$, Esther Beltran ${ }^{1}$, Antonio Fernando Bertachini de Almeida Prado ${ }^{5}$, Julie Castillo-Rogez ${ }^{6}$, William Grundy ${ }^{7}$, David Minton ${ }^{8}$, Arun Misra ${ }^{9}$, Jose-Luis Ortiz ${ }^{10}$, Noemi Pinilla-Alonso ${ }^{1}$, Darin Ragozzine $^{11}$, Diogo Merguizo Sanchez ${ }^{5}$, Gal Sarid ${ }^{12}$, and John Stansberry ${ }^{13}$
\end{abstract}

${ }^{1}$ Florida Space Institute, University of Central Florida, ${ }^{2}$ Planetary Science Institute, ${ }^{3}$ Arecibo Observatory, ${ }^{4}$ Southwest Research Institute, ${ }^{5}$ Instituto Nacional de Pesquisas Espaciais, ${ }^{6} \mathrm{Jet}$ Propulsion Laboratory, ${ }^{7}$ Lowell Observatory, ${ }^{8}$ Purdue University, ${ }^{9}$ McGill University, ${ }^{10}$ Instituto de Astrofísica de Andalucía, ${ }^{11}$ Brigham Young University, ${ }^{12}$ SETI Institute, ${ }^{13}$ Space Telescope Science Institute

*Contact: Julie.Brisset@ucf.edu; 407-823-6168

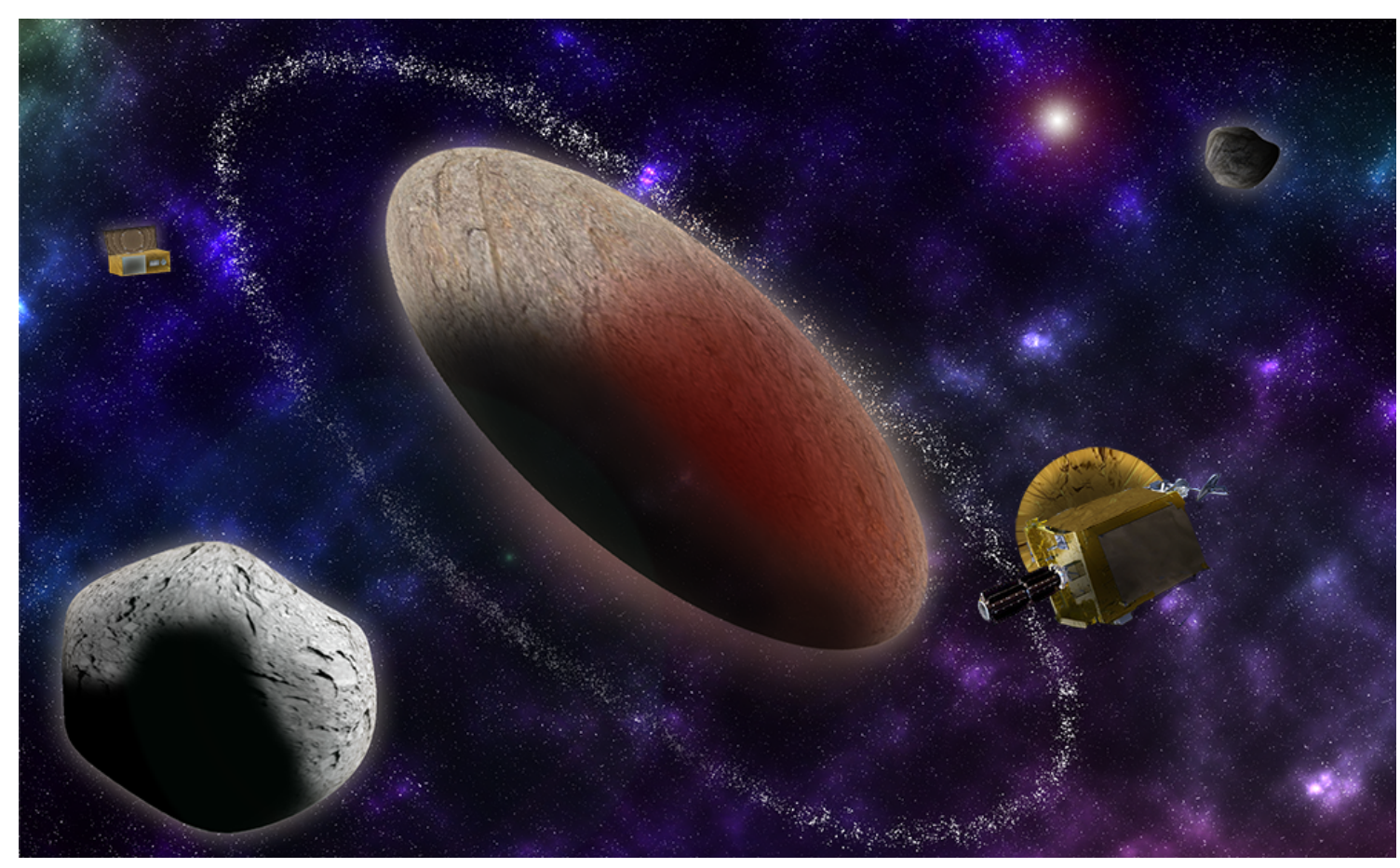




\section{Executive Summary}

Since its discovery in 2003, the dwarf planet Haumea has revealed itself to be one of the most intriguing bodies of the Solar System. It has an elongated shape and is spinning at an unusually fast rate of $4 \mathrm{~h}$. In addition, it is surrounded by a system of two satellites and a ring, and it is believed to be the parent body of the only collisional family in the Kuiper Belt known to date. The characteristics of the Haumea system and family have led to speculations on possible formation scenarios, including single and multiple collisions or rotational fission. It is also speculated that this dwarf planet could be the remnant core of a larger, differentiated Kuiper Blet Object (KBO), the mantle of which was disrupted by a giant impact. All these elements indicate that the Haumea system holds key information for several formation and evolution processes of bodies in the Kuiper Belt and the dwarf planet is now one of the most observed objects in that region of the Solar System.

This white paper summarizes the open questions about the Haumea system and family and advocates for their in-situ exploration, which would provide for invaluable insight into the history and on-going physical processes of the Kuiper Belt. Our recommendations for the next decade include:

- Mission Architecture Design, including detailed trajectory and transfer analysis to reach the Haumea system in reasonable flight times ( $\sim 15$ years or less);

- Technology development to allow for maximum science return at the Haumea system, including SmallSat bus and deployment technologies and;

- Mission support and science enhancement through Earth-based observations, numerical modeling, and laboratory work.

\section{Science Background}

Haumea was discovered in 2005, the same year as two other dwarf planets (Makemake and Eris). With dimensions of about 2,000 x 1,000 km [1], Haumea's size is on the same order as Pluto. As part of the family of dwarf planets in the Kuiper Belt, Haumea intrinsically carries information on formation and evolutionary processes of icy bodies, such as other KBOs, comets, Centaurs, as well as giant planet cores. In addition, Haumea has its own set of characteristics, making it a key target of study for advancing our understanding of the Solar System.

A fast spinner Haumea has a large rotational light-curve amplitude [2] due in part to its highly elongated shape (with axes ratios $\mathrm{b} / \mathrm{a}=0.73$ and $\mathrm{c} / \mathrm{a}=0.44$ [3]). In addition, Haumea has a very short rotation period of $\sim 4 \mathrm{~h}$ [2]. The stellar occultation produced by Haumea in 2017 allowed for an estimation of a density for the main body of $1.9 \mathrm{~g} / \mathrm{cm}^{3}$ [3], similar to Pluto's [4]. The axis ratios derived from this occultation are in disagreement with those expected for the hydrostatic equilibrium models [5], which would require a much higher density (given its short period and elongated shape). However, the currently measured value for the density could be explained by granular physics [6] or a differentiation of the body [1,7]. The highly detailed shape and topographic information achievable by a spacecraft visit would allow for the study of the behavior of icy material under unusual stress fields and what that implies for a body's shape, as well as the shapes of differentiated layers in the case of extreme rotation. It would reveal the bulk composition of the body and its mechanical strength, thus resolving the mystery of its nonhydrostatic equilibrium shape. Due to the short rotation period, the equatorial regions experience very low gravity environments, which could result in surface material loss or activity (as seen on asteroid 101955 Bennu by OSIRIS-Rex, [8]), a potential source of ring material. Detailed imaging of Haumea would reveal such processes and support a better understanding of activity detected around other fast-spinning icy bodies such as Centaurs (e.g., [9-11]). 
An unusual surface composition Haumea and some of its family members are unique in the Kuiper Belt with respect to their nearly pure and extremely strong water-ice spectral features $[12,13]$. Haumea's surface is known to be spectrally uniform [14], but photometrically varied due to a "Dark Red Spot" [15]. High spatial resolution spectroscopy and imaging will provide key information for understanding the origin and evolution of this surface heterogeneity. In addition, the strong and almost exclusive presence of water ice is sustaining a debate on the age of the surface and the processes leading to a depletion in other components, in particular carbon-rich materials. The strength of the water ice feature is also covering the potential presence of volatiles, such as $\mathrm{CH}_{4}$, which is a major constituent of other dwarf planets like Makemake and Eris. Only the detailed and spatially resolved spectroscopy of the surface offered by a spacecraft visit will allow for an in-depth understanding of Haumea's surface composition, providing clues to the reasons for the high amounts of water ice present compared to most other KBOs, and processes that can keep pure water ice at the surface of an aged body.

A system of rings and satellites Two satellites around Haumea were imaged using the Hubble Space Telescope and the Keck Observatory [16]. These satellites have regular orbital characteristics indicating that they formed in-situ in a proto-satellite disk around Haumea, but their current location is much further than standard tidal theories can explain [16-19]. Oblate Solar System bodies have been predicted to possess a stronger J2 coefficient in the secondary term in the gravitational potential [20]. An in-situ detailed measurement of Haumea's gravitational field may explain the odd orbits of rings and satellites.

The 2017 occultation of Haumea also revealed the presence of a ring around the dwarf planet [3]. Additional insight from high resolution imaging and satellite density characterization, as well as ring structural and compositional information will provide essential pieces to the puzzle of the formation of such a system. Clues to the ring-moon interactions will support setting constraints on the stability and evolution of the system. As rings have recently been discovered around other small icy bodies (the Centaurs Chariklo and potentially Chiron [21,22]), a visit to the Haumea system will also provide key information for the understanding of how rings can form and evolve around small icy bodies.

The Haumea family Small bodies are thought to have been highly affected by collisions that have left a clear imprint both on the properties of these objects and on their arrangement within the Solar System. In particular, collisional "families" can be identified in small body populations for Gy after the original collisions created them, based on clustering of orbital parameters. Such family members also frequently share similar surface properties $[23,24]$. This has been largely studied for objects in the main asteroid belt, where $\sim 140$ different families have been identified [24]. In the Kuiper Belt, only a single collisional family has been potentially identified up to date, and it is associated with Haumea [25]. Similarly to Haumea, these objects have strong indications of the presence of water ice in their spectra [25,26]. All of these water-rich KBOs, including Haumea, are spectrally neutral at optical wavelengths and dynamically close to each other, falling within a very small region of orbital elements space. It has been proposed that the icy mantle of the proto-Haumea was ejected during a catastrophic collision, resulting in a family of objects with very similar spectral features [25]. However, [27] and [28] show that the size and velocity dispersion distributions of Haumea family members are not consistent with a catastrophic collision. No final agreement between these models and observations have been achieved up to date, raising concerns about how well we understand collisions between icy bodies. A spacecraft visit to Haumea will provide unique information on the possibility of a large impact in Haumea's past. By observing the Haumea system as well as other members of the Haumea family, it will help state-of-the-art dynamical models to constrain possible formation 
scenarios for Kuiper Belt collisional families. In addition, a better understanding of the Haumea family could result in the detection of other families in the Kuiper Belt. The study of collisional families yields important information about the collisional processes that took place in the early Solar System, and thus, has important implications for our understanding of Solar System formation and evolution.

Haumea as a tracer for Kuiper Belt processes One of the most fundamental processes in the evolution of the Kuiper Belt is collisions between icy bodies, and little is still understood about the mechanics of such collisions. The study of collision tracers at the Haumea system, either on surfaces (craters, ice-coating), in the structure and composition of the ring-moon system, or in characteristics of another member of the Haumea family will provide truly unique insights into collision processes in the Kuiper Belt. In addition, detailed imaging of Haumea's and its satellites' surfaces will provide information about their structure and mechanical strength, which play an essential part in the outcome of collisions. As Haumea is part of the dwarf planet category, in a size range between small objects (asteroid, comets) and full-sized planets, its study will inform us on planet formation processes and their time scales in the early Solar System.

\section{In summary, Haumea is one of the most intriguing objects in the outer Solar System and a most informative target from which to learn about the different past and on-going physical processes taking place from the formation of the Kuiper Belt to the present day.}

\section{Advancing the State-of-the-Art Knowledge}

In the Solar System, small bodies such as asteroids and KBOs far outnumber any other type of bodies. While the relative closeness of asteroids allows for their systematic study, little is known about the small body population in the outer Solar System and the study of KBOs is an emerging field (with the first KBO other than Pluto only being discovered in 1992, [29]). KBOs are particularly interesting to study as they are the remnants of planet formation processes in the outskirts of our Solar System and retain unique information on the structure and composition of the solar nebula. The inspiring success of New Horizons (NH) and the overwhelming amount of data collected during the flybys of Pluto and Arrokoth have demonstrated how such a mission to the Kuiper Belt can significantly contribute to our understanding of processes involved in the formation and evolution the Solar System.

A mission to Haumea would have strong scientific synergies with several past, current, and future NASA missions and efforts. By exploring a new dwarf planet of the Solar System, it will support and enhance the outcome of other dwarf planet missions, such as Dawn at Ceres and New Horizons at Pluto. The study of Haumea's water-rich surface will allow for comparative planetology with water-rich bodies in the Solar System, such as some of the giant planets' moons: Triton and Uranian satellites, visited by Voyager 1; Enceladus, visited by Cassini; Europa, visited by Galileo and to be studied in more details by Europa Clipper. In addition, detailed imaging and spectroscopy of Haumea and its system will enhance the value of NASA telescope studies of small icy bodies (Hubble Space Telescope, Spitzer Space Telescope, and the future James Webb Space Telescope), which are able to image numerous objects, albeit at much lower spatial resolutions.

The next decade is the ideal time to consider a mission to the Haumea dwarf planet in the Kuiper Belt: we are at a turning point in our understanding of the role and interactions of small icy bodies in our Solar System's history; numerous observations and an occultation of Haumea have provided for a solid knowledge base on the target, thus allowing maximizing scientific return and reducing risks associated with a space mission; and New Horizons has demonstrated that such a distant world is within reach of a space mission. 


\section{Science Questions}

A detailed study of the Haumea system will allow the access to a significant amount of information on KBO formation and evolution processes as well as on the effects of large-scale collisions involving icy bodies. In Table 1, we present a list of open questions about KBOs and the Haumea system in particular, which can be addressed by a detailed study of the Haumea system.

Table 1: Major science questions that can be addressed by the study of the Haumea system.

What are KBO formation and evolution processes?

- How does the presence of ices influence the accretion and differentiation processes?

- What is the internal structure of KBOs? How does it influence their shape and spin?

- How do ring-moon systems form and evolve around small icy bodies?

- How do icy surfaces weather in the Kuiper Belt?

- Is surface activity common among KBOs?

- Are dynamical families common in the Kuiper Belt?

How do icy collisions shape these distant worlds?

- How common are collisions in the past and present Kuiper Belt?

- How does the presence of ices influence the cratering/excavation processes?

- Can icy collisions lead to global or partial resurfacing?

- Can KBO collisions be at the origin of elongated body shapes, fast spin rates, and ring-moon systems?

How did the Haumea system form and evolve?

- What are Haumea's physical characteristics? (density, composition, surface features, etc.)

- What are the rings and satellites' characteristics in the Haumea system? (satellite density, composition, surface features, ring particle size, number density, composition, ring structure and features, etc.)

- What are the characteristics of Haumea's KBO environment? (dust and radiation)

- What are the characteristics of the Haumea family members?

\section{Recommendations for the Next Decade}

The outstanding science questions above can be addressed within the next decade through the study of mission architectures, supported by research and analysis. This section explores what it would take to advance the solar system science enabled by the study of the Haumea system over the coming decade.

\section{Need for a Concept Mission Study}

Most of the challenges associated with a mission to Haumea are caused by the large distance between the dwarf planet and the Earth. Currently, Haumea is at about 50 AU from Earth, after passing its aphelion in 1992. Figure 1 shows the distance from Earth to Haumea from now until 2060 and a time frame that can include the arrival date of a future mission there. This large distance requires long travel times and high spacecraft velocities. At the same time, communication links with the spacecraft upon approach of the target are slow (several hours oneway), as well as much weaker than for closer targets. The large distance from the Sun prohibits the possibility of powering the spacecraft via solar power. In addition, the detected presence of rings around the dwarf planet indicates a higher risk for an active dust environment, which can cause damage to the spacecraft. A concept mission study is needed to address the following:

New Horizon Heritage A mission to Haumea will greatly benefit from the recent spaceflight experience acquired with $\mathrm{NH}$ to Pluto and the Kuiper Belt. An encounter with Haumea after 


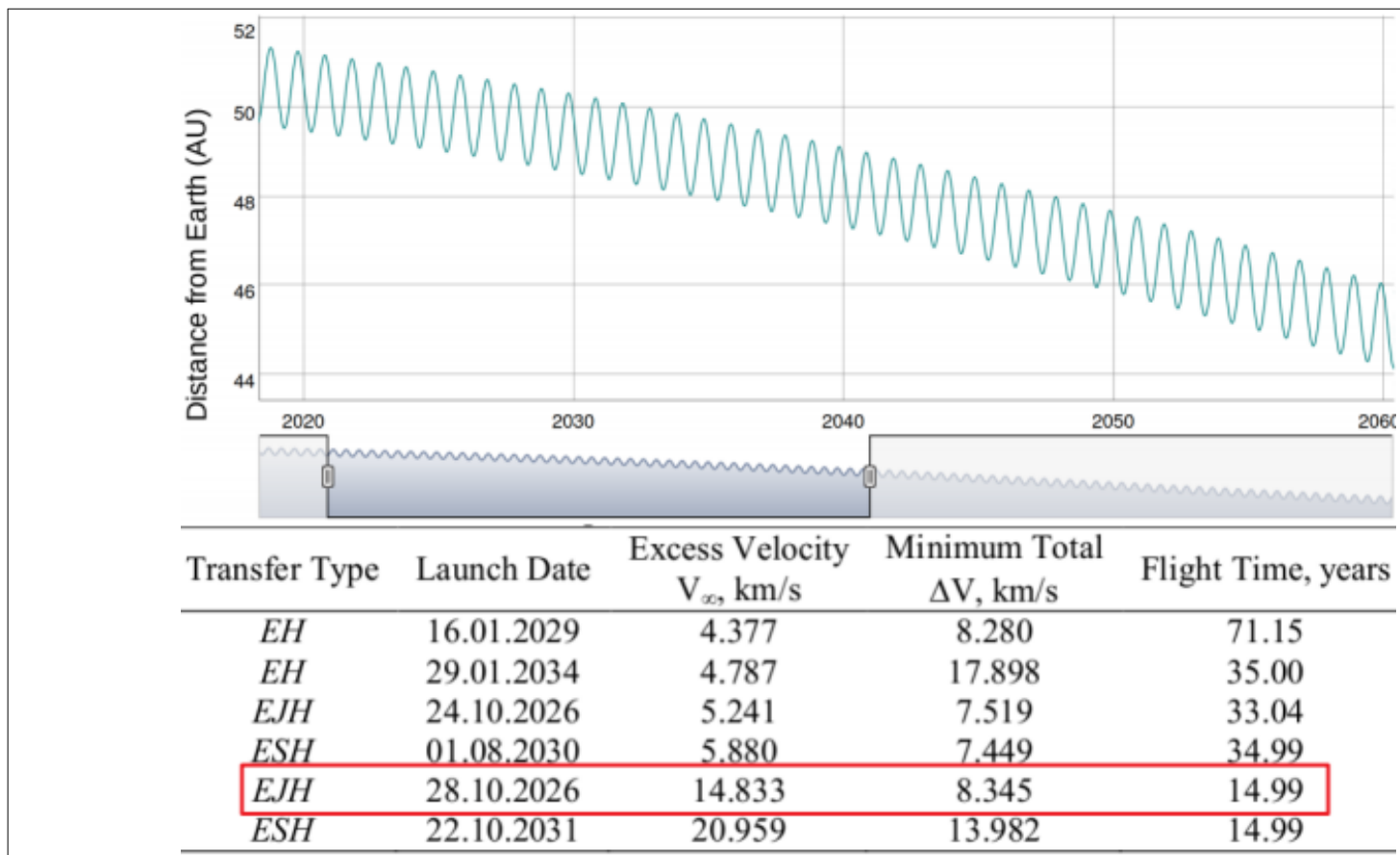

Figure 1: Reaching Haumea: (top) Distance from Earth to Haumea in the next decades. Credit: theskylive.com; (bottom) Optimal trajectories for several types of transfer for a mission to Haumea. E: Earth; H: Haumea; J: Jupiter; S: Saturn. Credit: Sanchez, et al., 2014.

realistic mission travel times of 15 years can happen at about 47 AU (Figure 1 bottom table, $[30,31])$, which is in the same range as the Arrokoth flyby by NH. Thanks to NH, a single flyby at such large distances, for target bodies of similar or smaller sizes than Haumea, has now been proven to be a successful mission concept. Haumea's fast rotation rate ( $4 \mathrm{~h},[2])$ would also allow for a simple flyby to observe most of its surface.

Including SmallSats The simple flyby mission concept can be expanded to the inclusion of detachable units. Scientific return enhancements through detachable spacecraft units are a common feature of space missions and probes. Examples are impactors (e.g. Deep Impact at comet Temple 1 in 2005), small landers (e.g. Hayabusa-2 at asteroid Ryugu in 2018), or communication relays (e.g. the MarCO CubeSats at Mars in 2019). While the current CubeSat technology level would not support a direct communication link to Earth, a small detachable unit deployed in the vicinity of Haumea could be operated in conjunction with the main spacecraft as a mothership. For a deployment before the flyby for example, additional imaging of the Haumea system from a second vantage point will allow for increased data collection of satellites and rings, and has therefore potential to significantly increase the flyby's science return.

Targets of Opportunity Following NH's example, the cruise phase of a spacecraft on its way to Haumea can be used for observing other Solar System objects from the closer vantage point of the traveling spacecraft. This can be implemented by the inclusion of a long-range imager of the type of LORRI (Long-Range Reconnaissance Imager) in the instrument suite of the main spacecraft. [30] show that an optimized trajectory to Haumea using a Jupiter gravity assist maneuver can allow for the flyby of several asteroids in the main belt. A gravity assist maneuver at Jupiter would also allow for additional data collection at the gas giant, while being an opportunity to develop a flyby observation package and execute it prior to arriving at Haumea. In addition, one of the Haumea family members, 2005 CB79, is in the vicinity of Haumea and can be observed in more detail during the time of approach. Information about this $\mathrm{KBO}$, in 
particular more detailed surface composition data, will be of great interest in the discussion on whether this family member, which is dynamically close to Haumea, might actually have been formed together with the dwarf planet itself. Noticeably, another dwarf planet, Makemake, will also be in Haumea's vicinity in the 2040's and 50's. Makemake has a similar size to Pluto and is traveling on an orbit that is stable at time scales of the age of the Solar System. It has one confirmed satellite and a suspected tenuous atmosphere [26,32]. A mission to Haumea would be a great opportunity to collect more data on this other dwarf planet.

\section{Technology Development}

A mission to Haumea would strongly benefit from flight heritage of $\mathrm{NH}$, both for spacecraft bus functions (power, telecommunications), and for instrument concepts. Technology developments of interest for such a future mission include, for example, Small-/CubeSat capabilities allowing them to perform autonomous operations at such large heliocentric distances.

\section{Research and Analysis}

Observational Data Collection and Analysis Stellar occultations are one of the best methods by which to study physical properties of KBOs: sizes can be measured to km-level accuracy, atmospheres can be probed to the level of a few microbars, and ring systems and satellites can be discovered and characterized. The 2017, a stellar occultation by Haumea returned unprecedented information [3]. Additional occultation observations by Haumea are needed to further refine the shape model, study short-term evolution of the ring system, and search for (or rule out) any localized atmosphere. Occultation observations of other large KBOs will contribute to our understanding of formation and evolution processes of the entire population.

Observations with the JWST will also provide some insight into the Haumea collisional family. Guaranteed Time Observations (GTO) 1191 is planning to study the surface composition of several of its members as well as other candidates to be part of the family. In addition, GTO 1273 is planning to study the rotational variability of Haumea using spectroscopic data from NIRSpec and MIRI. Comparison between data from NIRSpec and MIRI will help to constrain the Haumea surface composition. JWST observations will contribute valuable information on the Haumea system, in particular on surface composition thanks to the very high spectral (albeit not spatial) resolution of its on-board spectrometer.

Numerical Modeling Investing in adequate numerical efforts will provide for unique insights into the formation of the Haumea system, as well as physical processes that took place in the Kuiper Belt. Our knowledge of the Kuiper Belt history can be advanced by the means of largescale dynamical simulations of the KBO population since its formation. In addition, we can learn about the interior structure of KBOs and the formation of collisional families, rings, and satellites using orbital dynamics simulations of particulate mass upon collision, including ring capture and evolution. Small-scale Discrete Element Method (DEM) simulations of multi-particle environments will help understand KBO ring evolution and stability.

Laboratory Work In order to support a better understanding of $\mathrm{KBO}$ formation and evolution, and in particular of the Haumea system, a better understanding of collisions and soil mechanics when ices are present in the target material will be required. Such data is required to calibrate and validate numerical models of large-scale collision event or full granular body evolution. Our knowledge of icy material behavior can be advanced by the means of laboratory experiments and measurements. Cryogenic facilities, high-speed impact facilities, and microgravity platforms can be used to achieve relevant data collection. In addition, the weathering of icy surfaces can be studied using laboratory spectroscopy on cryogenic samples, previously irradiated to simulate space weathering in the Kuiper Belt. 
Exploring the Haumea System

\section{References}

[1] E.T. Dunham, S.J. Desch, L. Probst, Astrophys. J. 877 (2019) 41.

[2] D.L. Rabinowitz, K. Barkume, M.E. Brown, H. Roe, M. Schwartz, S. Tourtellotte, C. Trujillo, ApJ 639 (2006) 1238.

[3] J.L. Ortiz, P. Santos-Sanz, B. Sicardy, G. Benedetti-Rossi, D. Bérard, N. Morales, R. Duffard, F. Braga-Ribas, U. Hopp, C. Ries, others, Nature 550 (2017) 219.

[4] S.A. Stern, W.M. Grundy, W.B. McKinnon, H.A. Weaver, L.A. Young, Annu. Rev. A\&A 56 (2018) 357-392.

[5] S. Chandrasekhar, Ellipsoidal Figures of Equilibrium, Yale University Press New Haven, 1969.

[6] K.A. Holsapple, Icarus 187 (2007) 500-509.

[7] N. Rambaux, F. Chambat, J. Castillo-Rogez, D. Baguet, in: AAS/DPS Meet. Abstr. 48, 2016.

[8] D.S. Lauretta, C.W. Hergenrother, S.R. Chesley, J.M. Leonard, J.Y. Pelgrift, C.D. Adam, M. Al Asad, P.G. Antreasian, R.-L. Ballouz, K.J. Becker, others, Science (80-. ). 366 (2019).

[9] J.M. Bauer, Y.R. Fernández, K.J. Meech, Publ. Astron. Soc. Pacific 115 (2003) 981.

[10] E.M. Epifani, P. Palumbo, M.T. Capria, G. Cremonese, M. Fulle, L. Colangeli, A\&A 460 (2006) 935-944.

[11] S. Fornasier, D. Lazzaro, A. Alvarez-Candal, C. Snodgrass, G.P. Tozzi, J.M. Carvano, Y. JiménezTeja, J.S. Silva, D.M. Bramich, A\&A 568 (2014) L11.

[12] K.M. Barkume, M.E. Brown, E.L. Schaller, Astrophys. J. Lett. 640 (2006) L87.

[13] C.A. Trujillo, M.E. Brown, K.M. Barkume, E.L. Schaller, D.L. Rabinowitz, Astrophys. J. 655 (2007) 1172.

[14] N. Pinilla-Alonso, R. Brunetto, J. Licandro, R. Gil-Hutton, T.L. Roush, G. Strazzulla, A\&A 496 (2009) 547-556.

[15] P. Lacerda, Astron. J. 137 (2009) 3404.

[16] D. Ragozzine, M.E. Brown, Astron. J. 137 (2009) 4766.

[17] M. Ćuk, D. Ragozzine, D. Nesvornỳ, Astron. J. 146 (2013) 89.

[18] D.M. Hastings, D. Ragozzine, D.C. Fabrycky, L.D. Burkhart, C. Fuentes, J.-L. Margot, M.E. Brown, M. Holman, Astron. J. 152 (2016) 195.

[19] A.C. Quillen, A. Kueter-Young, J. Frouard, D. Ragozzine, Mon. Not. R. Astron. Soc. 463 (2016) $1543-1553$.

[20] A.M. Hofmeister, R.E. Criss, E.M. Criss, Planet. Sp. Sci (2017).

[21] F. Braga-Ribas, B. Sicardy, J.L. Ortiz, C. Snodgrass, F. Roques, R. Vieira-Martins, J.I.B. Camargo, M. Assafin, R. Duffard, E. Jehin, others, Nature 508 (2014) 72.

[22] J.L. Ortiz, R. Duffard, N. Pinilla-Alonso, A. Alvarez-Candal, P. Santos-Sanz, N. Morales, E. Fernández-Valenzuela, J. Licandro, A.C. Bagatin, A. Thirouin, A\&A 576 (2015) A18.

[23] J.R. Masiero, F.E. DeMeo, T. Kasuga, A.H. Parker, Asteroids IV (2015) 323-340.

[24] D. Nesvornỳ, M. Brož, V. Carruba, others, Asteroids IV (2015) 297-321.

[25] M.E. Brown, K.M. Barkume, D. Ragozzine, E.L. Schaller, Nature 446 (2007) 294.

[26] E.L. Schaller, M.E. Brown, Astrophys. J. Lett. 684 (2008) L107.

[27] R. Pike, D. Ragozzine, B. Proudfoot, S. Maggard, M. Alexandersen, Ossos Core Team, in: AAS/DPS Meet. Abstr. \#50, 2018, p. 302.02.

[28] B. Proudfoot, D. Ragozzine, ArXiv Prepr. ArXiv1904.00038 (2019).

[29] D. Jewitt, J. Luu, Nature 362 (1993) 730-732.

[30] D. Sanchez, A.F. Prado, A. Sukhanov, T. Yokoyama, in: SpaceOps 2014 Conf., 2014, p. 1639.

[31] D.M. Sanchez, A.A. Sukhanov, A. Prado, Rev. Mex. Astron. y Astrofisica 55 (2019) 39-54.

[32] M.E. Brown, K.M. Barkume, G.A. Blake, E.L. Schaller, D.L. Rabinowitz, H.G. Roe, C.A. Trujillo, Astron. J. 133 (2006) 284. 